\title{
THE FORM OF ENERGY GAP AND CRITICAL TEMPERATURE OF SUPERCONDUCTOR IMPLIED BY FLUCTUATIONS OF DENSITY OF STATES
}

\author{
M. Gladysiewicz, R. Gonczarek and M. Mulak \\ Institute of Physics, Wrocław University of Technology \\ Wybrzeże Wyspiańskiego 27, 50-370 Wrocław, Poland
}

(Received October 21, 1999; revised version February 8, 2000)

\begin{abstract}
The s-paired BCS superconductor with the electron density of states depending on energy in the vicinity of Fermi level is considered. The existence of such fluctuations of electron density of states may be referred to Van Hove singularities widely discussed in the literature. In the paper three types of small fluctuations of the electron density of states introduced about its a verage background's value are analysed: Lorentzian, triangle, and logarithmic ones. In order to calculate numerically the energy gap and critical temperature, the formalism of parametric BCS gap equations is applied. The positive fluctuations (peaks) correspond to the increase in occupied states number inducing a higher critical temperature. On the other hand, negative fluctuations, which decrease the number of occupied states, lead to a lower critical temperature. Such decrease in critical temperature can lead, at a specific choice of parameters, to the decease in superconductivity. The presence of fluctuations finds reflection in the shape of energy gap as a function of temperature. In the vicinity of $T=0$ an abrupt decrease or increase in the energy gap to the original BCS value, according to the sign of the fluctuation, is observed. In turn, the fluctuations do not change the behaviour of the energy gap near $T_{\mathrm{c}}$.
\end{abstract}

PACS numbers: $74.20,-\mathrm{Z}$

\section{Introduction}

Singularities of the electron density of states (EDOS) and their influence on superconducting properties have been recently widely discussed in the literature within the Van Hove scenario, e.g. $[1,3,4]$. The reason for the existence and mechanisms of high-temperature superconductivity is not clear up today and it 
seems to be possible that fluctuations of EDOS may turn to be crucial in its explanation. Within the Van Hove scenario we try to answer a profound question, how the physics of interacting electron liquid is modified by presence of Van Hove singularities near the Fermi level, which were originally classified in two and three dimensions by Leon Van Hove [5].

From the point of view of cuprates physics the two-dimensional singularity, i.e. a saddle point, which is associated with a crossover from electron-like to hole-like conduction, might be extremely important. In the paper [3] the density of states with a superposition of one-dimensional extremum and two-dimensional saddle point (connected with a logarithmic divergence) has been studied and an analytical approximation for the BCS gap equation within the weak coupling theory was proposed. As a result the values of $T_{\mathrm{c}}$ between $40 \mathrm{~K}$ and $140 \mathrm{~K}$ as well as the isotope parameter between 0.18 and 0.28 were established. In turn, in the paper [4] the logarithmic EDOS with Coulomb repulsion was investigated when the electronic interaction was renormalised to obtain the effective repulsive pseudopotential. In this case a high critical temperature was also achieved. In the review [1] many theoretical and experimental approaches associated with Van Hove scenario are discussed. According to them a reasonable comprehension of the high temperature superconductivity phenomenon could be related to some unusual properties of cuprates with singularities in EDOS.

Before the discovery of cuprates an empirical rule was stated that the best way to find out a high temperature superconductor was to look for a material with a peak in EDOS. Recently, Mitrovic and Carbotte have proposed some interesting models of EDOS with peaks close to $E_{\mathrm{F}}$ for $A 15$ compounds in order to explain the anomalous behaviour of their normal and superconducting states, respectively $[6,7]$. They have considered fluctuations of EDOS in the Lorentzian and triangle shapes analysing the problem within the isotropic approximation. Band structure calculations have confirmed the possibility of such sharp peaks in EDOS for materials with the $A 15$ crystal structure [8], as well as some recent angular resolved photoemission spectroscopy (ARPES) experiments have shown that many high temperature superconductors possess a sharp peak in EDOS near Fermi energy [1].

In this paper we model such simple fluctuations and the numerically calculated critical temperature together with the energy gap dependence vs. temperature. The starting point of further calculations is the BCS gap equation.

\section{The model}

Let us consider an $s$-paired BCS superconductor with a small fluctuation added to constant background's EDOS $N(0)$. The modified EDOS has the form

$$
N(\xi, \text { parameters })=N(0)(1+\text { fluctuation })=N(0) N_{1}(\xi, \text { parameters }) .
$$

Below, we focus our interests on three particular types of fluctuations:

- Lorentzian type:

$$
N_{1}(\xi, B, R, x, n)=1+\sum_{i=1}^{n} \frac{x_{i}}{1+\left[\left(\xi-R_{i}\right) / B_{i}\right]^{2}} ;
$$


The above equation represents a superposition of Lorentzian peaks on EDOS, originally discussed in $[6,7]$. Let us emphasise that the interesting thermodynamic properties of the system with such Lorentzian EDOS were calculated in the paper [9].

- Triangle type:

$$
N_{1}(\xi, B, R, x, n)=1+\sum_{i=1}^{n}\left(x_{i}-\frac{\left|\xi-R_{i}\right|}{B_{i}}\right) \Theta\left(x_{i}-\frac{\left|\xi-R_{i}\right|}{B_{i}}\right)
$$

Here EDOS has $n$ triangle peaks near the Fermi level.

- Logarithmic type:

$$
N_{1}(\xi, B, R, x, n)=1+\sum_{i=1}^{n} x_{i} \ln \frac{2 B_{i}}{\left|\xi-R_{i}\right|} \Theta\left(\frac{2 B_{i}}{\left|\xi-R_{i}\right|}-1\right)
$$

where the fluctuation is represented by the logarithmic divergences localised on constant EDOS. This type of fluctuation may be often found in high temperature superconductors $[1,2]$.

In the postulated forms of EDOS $\xi$ is the energy referred to the Fermi level, $x_{i}$ the height of the fluctuation, Eqs. (2) and (3) or the growth rate of the singularity (Eq. (4)), $B_{i}$ - its half-width, $R_{i}$ - the energy distance (shift) of the fluctuation from the Fermi level and $n$ is the number of peaks or singularities under consideration. Since only small fluctuations are taken into account the average number of quasiparticles $N$ is preserved and constant

$$
\int_{-\omega_{D}}^{\omega_{D}} N(\xi, B, R, x, n) \simeq 2 \omega_{D} N(0)=N,
$$

where $B, R$, and $x$ denote $n$-dimensional vectors of $B_{i}, R_{i}$, and $x_{i}$, respectively.

We begin with more general form of the BCS energy gap equation taking EDOS as non-symmetric with respect to the Fermi level

$$
1=\frac{\lambda}{2} \int_{-\omega_{D}}^{\omega_{D}} \frac{\mathrm{d} \xi}{\sqrt{\xi^{2}+\Delta^{2}}} N_{1}(\xi, B, R, x, n) \tanh \frac{\sqrt{\xi^{2}+\Delta^{2}}}{2 T},
$$

where $\lambda=N(0) g_{0}$ is the pairing parameter. Introducing the symbols (cf. [10-13])

$$
u=\frac{\xi}{2 T}, \quad \tau=\frac{\Delta(T)}{2 T}, \quad r=\frac{R}{2 T}, \quad b=\frac{B}{2 \Delta(0)}
$$

and integrating Eq. (6) by parts with two different integral functions, the gap equation can be written in the following form:

$$
\begin{aligned}
\frac{1}{\lambda}= & \ln \left(\frac{2 \omega_{D}}{\Delta(T)}\right)-\frac{1}{2} \int_{-\infty}^{\infty} \mathrm{d} u\left[\ln \left(u+\sqrt{u^{2}+\tau^{2}}\right)-\ln \tau\right] \\
& \times \frac{\partial}{\partial u}\left[N_{1}\left(u, b, r, x, n, \frac{T}{\Delta(0)}\right) \tanh \sqrt{u^{2}+\tau^{2}}\right]
\end{aligned}
$$

and consequently 


$$
\begin{aligned}
\frac{1}{\lambda}= & \ln \left(\frac{\omega_{D}}{T}\right)-\frac{1}{2} \int_{-\infty}^{\infty} \mathrm{d} u \frac{|u|}{u} \ln \left(|u|+\sqrt{u^{2}+\tau^{2}}\right) \\
& \times \frac{\partial}{\partial u}\left[N_{1}\left(u, b, r, x, n, \frac{T}{\Delta(0)}\right) \tanh \sqrt{u^{2}+\tau^{2}}\right] .
\end{aligned}
$$

In all our calculations the following conditions are assumed:

$$
N_{\perp}\left(u, b, r, x, n, \frac{T}{\Delta(0)}\right)=1 \text { and } \frac{\partial N_{1}}{\partial u}=0
$$

if $u$ tends to the limit of the integration interval $\left(|u| \rightarrow \omega_{D} / 2 T\right)$, which is often replaced by infinity. The above procedure is fully consistent with the assumption given by Eq. (5).

Note that in Eqs. (8) and (9) a new parameter $T / \Delta(0)$ has been introduced. Now, the limits $T=0(\tau=\infty)$ and $T=T_{\mathrm{c}}(\tau=0)$ are investigated and after some algebraic transformations one may obtain, respectively

$$
\frac{1}{\lambda}=\ln \frac{2 \omega_{D}}{\Delta(0)}
$$

and

$$
\frac{1}{\lambda}=\ln \frac{\omega_{D}}{T_{\mathrm{c}}}-\int_{-\infty}^{\infty} \mathrm{d} u \frac{|u|}{u} \ln |2 u| \frac{\partial}{\partial u}\left[N_{1}\left(u, b, r, x, n, \frac{T_{\mathrm{c}}}{\Delta(0)}\right) \tanh |u|\right] .
$$

It is convenient to introduce the following substitutions:

$$
X(\tau)=\pi \mathrm{e}^{-c} \frac{T}{\Delta(0)}=\frac{T}{T_{\mathrm{co}}}, \quad Y(\tau)=\pi \mathrm{e}^{-c} \frac{\Delta(T)}{\Delta(0)}=\frac{\Delta(T)}{T_{\mathrm{c} 0}},
$$

which are useful for numerical calculations. Let us emphasise that the parameter $\tau$ takes values from zero to infinity.

\section{Lorentzian EDOS}

Below, Eq. (11) with (12) is solved numerically by the perturbational method for the fluctuation given by (2). Dealing with Eqs. (8) and (10) we employ the efficient numerical algorithm

$$
\begin{array}{r}
Y(\tau)=\pi \mathrm{e}^{-c} \exp \left\{-\frac{1}{2} \int_{-\infty}^{\infty} \mathrm{d} u \ln \left[\left(u+\sqrt{u^{2}+\tau^{2}}\right)-\ln \tau\right]\right. \\
\left.\times \frac{\partial}{\partial u}\left[\left(1+\sum_{i=1}^{n} \frac{x_{i}}{1+\left(\frac{u-r_{i}}{b_{i}}\right)^{2} \frac{T}{\Delta(0)}}\right) \tanh \sqrt{u^{2}+\tau^{2}}\right]\right\},
\end{array}
$$

where

$$
X(\tau)=\frac{Y(\tau)}{2 \tau} .
$$

Equations (13), (14) turn to be very convenient to calculate the energy gap dependence vs. temperature.

In turn, to derive the new critical temperature, based on Eqs. (9) and (11), we apply the algorithm as follows: 
$t=\frac{T_{\mathrm{c}}}{\Delta(0)}=\frac{1}{2} \exp \left\{\frac{1}{2} \int_{-\infty}^{\infty} \mathrm{d} u \ln |2 u| \frac{\partial}{\partial u}\left[1+\sum_{i=1}^{n} \frac{x_{i}}{1+\left(\frac{u-r_{i}}{c_{i}}\right)^{2}}\right] \tanh (u)\right\}$

where

$$
\frac{T_{\mathrm{c}}}{T_{\mathrm{c} 0}}=\pi \mathrm{e}^{-c} t, \quad c_{i}=\frac{b_{i}}{t} .
$$

Firstly, let us consider the case of a single Lorentzian peak. The energy gap temperature dependence is plotted in Figs. 1, 2, and 3. Near $T \rightarrow 0$ one may observe its rapid change. These results remain in agreement with the fundamental BCS relation $\Delta(0) / T_{\mathrm{c}}$.

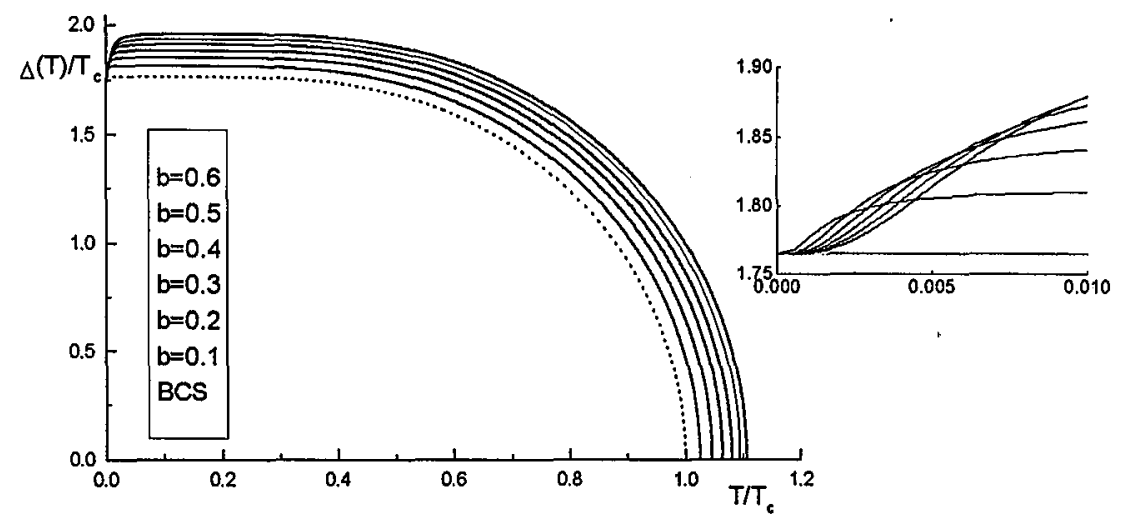

Fig. 1. The exemplary forms of the energy gap calculated for the Lorentzian EDOS vs. reduced temperature for a few chosen values of $b$. Parameters $x$ and $r$ are fixed and $x=0.1, r=0$. Parameter $b$ takes the values: $0.6,0.5,0.4,0.3,0.2,0.1$. The original BCS curve is represented by the dotted line $(b=0)$.

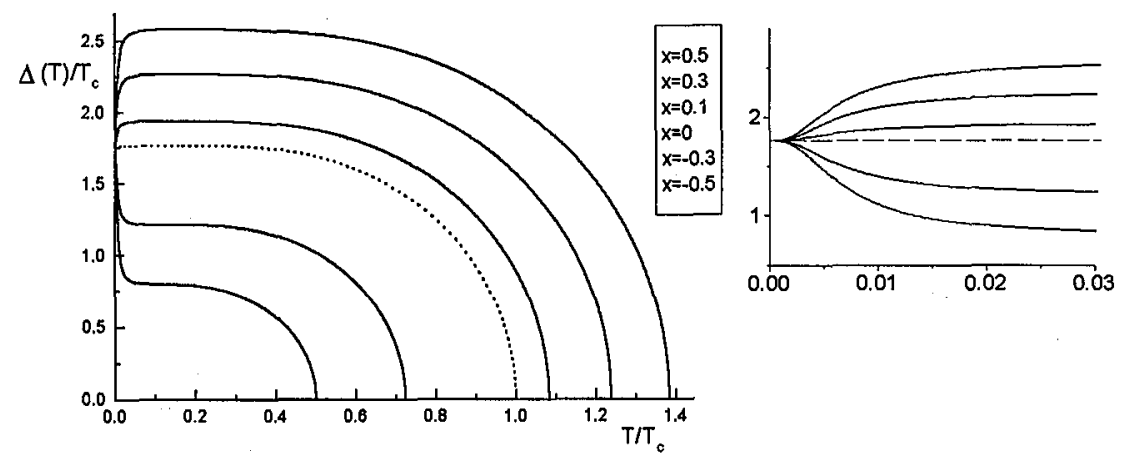

Fig. 2. The exemplary forms of the energy gap calculated for the Lorentzian EDOS vs. reduced temperature for a few chosen values of $x$. Parameters $b$ and $r$ are fixed and $b=0.5, r=0$. Parameter $x$ takes the values: $0.5,0.3,0.1,0,-0.3,-0.5$. The dotted line represents the case of $x=0$ (the BCS curve). 


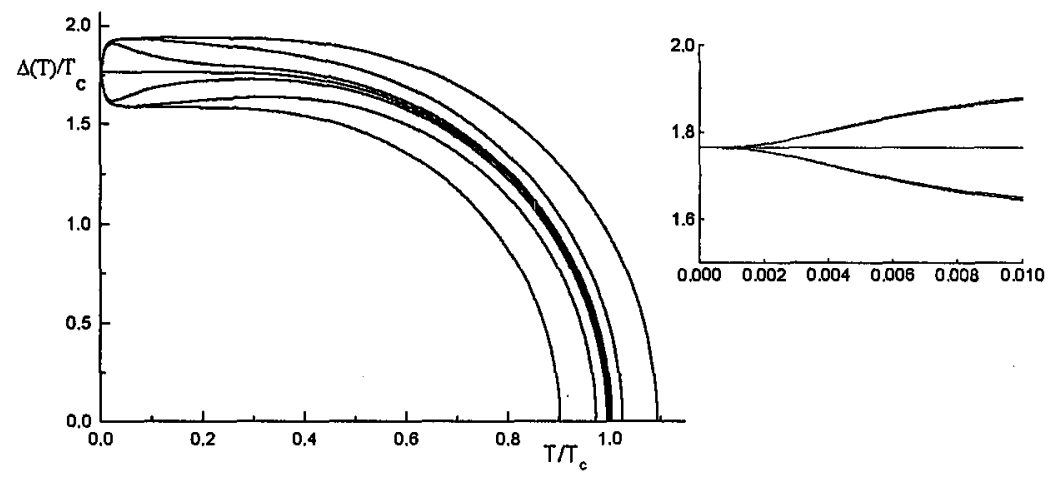

Fig. 3. The exemplary forms of the energy gap calculated for the Lorentzian EDOS vs. reduced temperature for a few chosen values of $r$. Parameters $b$ and $x$ are fixed and $b=0.5$. For the upper three curves $x=0.1$. Parameter $r$ takes the following values: 0 , 6,30 . The next line corresponds to the original BCS curve $(x=0)$. Finally, the lower three curves correspond to $x=-0.1$. Parameter $r$ is equal to $30,6,0$, respectively.

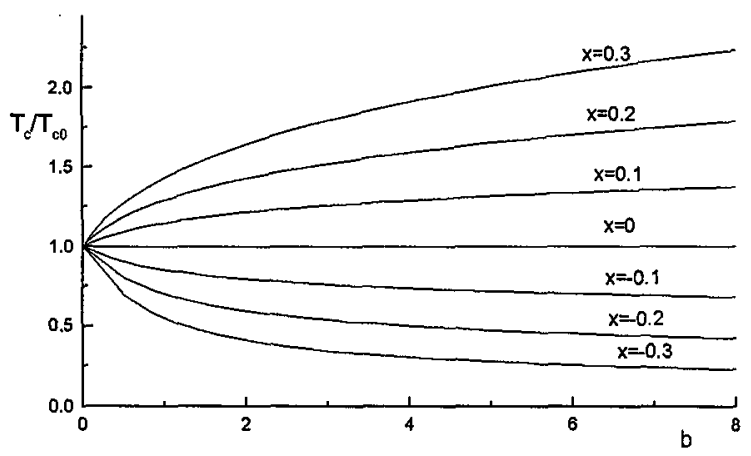

Fig. 4. Reduced critical temperature calculated for the Lorentzian EDOS vs. $b$ parameter. Parameter $r=0$ and $x$ takes the values: $0.3,0.2,0.1,0,-0.1,-0.2,-0.3$.

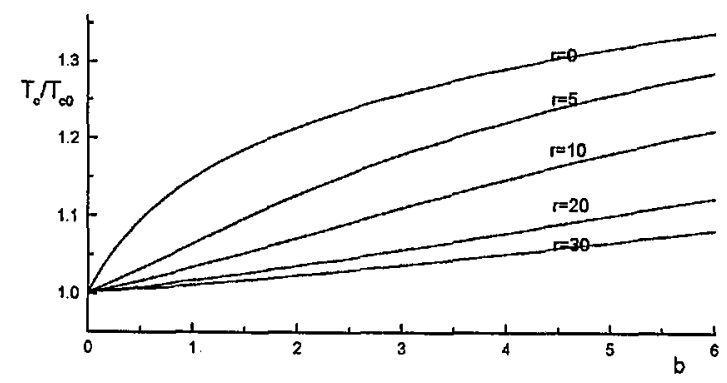

Fig. 5. Reduced critical temperature calculated for the Lorentzian EDOS vs. $b$ parameter. Parameter $x=0.1$ and $r$ takes the values: $0,5,10,20,30$. 
Let us emphasise that the appearance of the plateau of the energy gap in the very small region close to $T=0$, which one can observe in the insets of Figs. 1, 2, 3, is due to the relation between the energy gap and heat capacity. As it is shown in [10] the heat capacity $C \sim \partial \Delta / \partial T$, so in the obtained results $C \rightarrow 0$ at $T \rightarrow 0$, and the third law of thermodynamics is also fulfilled.

The curves corresponding to different $b$ parameters, at fixed $x$ and $r$, are depicted in Fig. 1. In turn, the curves for different $x$ parameters but now at fixed $b$ and $r=0$, are given in Fig. 2, where they are distributed symmetrically with respect to the classical BCS curve. If the parameter $r$ is not equal to 0 then the energy gap vs. temperature has an extremum, a maximum for positive $x$ and a minimum for negative $x$, respectively (see Fig. 3).

It is clearly seen that the basic parameters of fluctuation $x$ and $b$ have a strong and straightforward influence on the value of critical temperature, which is shown in a direct manner in Fig. 4. The critical temperature grows up at positive $x$ and large $b$, and goes down at negative $x$, when even small $T_{\mathrm{c}}$ could be obtained when $b$ is enough large. In the below Subsection 3.1. some intriguing analytical results, corresponding just to this case, are briefly presented. On the other hand, the influence of the parameter $r$ on $T_{\mathrm{c}}$ is summarised in.Fig. 5.

\subsection{Decay of superconductivity}

In this subsection the analytical calculation to prove that the critical temperature may go to zero in the model with negative fluctuation is sketched. Using the following formula:

$$
\tanh (u)=2 u \sum_{i=1}^{\infty} \frac{1}{\pi^{2}(k+1 / 2)^{2}+u^{2}},
$$

after integration we find

$$
\begin{aligned}
\ln \frac{t}{t_{0}} & =\sum_{i=1}^{\infty} \frac{x_{i} b_{i}}{t} \sum_{k=1}^{\infty}\left\{\frac{\pi}{\left[\frac{\pi}{2}(2 k+1)+b_{i} / t\right]^{2}+c_{i}}\right. \\
& \left.+\frac{2 b_{i}}{t(2 k+1)\left[\left(\frac{\pi}{2}(2 k+1)+b_{i} / t\right)^{2}+c_{i}^{2}\right]}\right\},
\end{aligned}
$$

where $t_{0}=T_{\mathrm{c} 0} / \Delta(0)=\pi \mathrm{e}^{-c}$. Equation (18), in the limits of $c_{i} \rightarrow 0, t \rightarrow 0$, takes the following form:

$$
\ln \frac{t}{t_{0}}=\sum_{i=1}^{n} x_{i} \sum_{k=1}^{\infty}\left(\frac{1}{k+\frac{1}{2}}-\frac{1}{k+\frac{1}{2}+b_{i} / \pi t}\right),
$$

from which one can finally get

$$
\left(1+\sum_{i=1}^{n} x_{i}\right) \ln \frac{t}{t_{0}}=\sum_{i=1}^{n} x_{i}\left(\ln 4 b_{i}-\frac{\pi t}{b_{i}}\right) .
$$

Equation (20) shows that for some specific negative $x_{i}$ parameters and $b_{i}$ which are always positive, the critical temperature, $T_{\mathrm{c}}=\Delta(0) t$, may attain very small values, dropping to zero in particular cases, which clearly corresponds to destruction of 
superconductivity. This conclusion remains entirely in agreement with our previous numerical results. It indicates that the fluctuation of EDOS can also decrease the transition temperature though this effect is not specifically investigated.

\section{Triangle EDOS}

Now, analogously to Section 3, the triangle form of EDOS (see Eq. (3)) can be put into Eq. (8), and after parameterization according to (7), one may obtain a numerical algorithm to find the energy gap dependence vs. temperature in the form

(i)

$$
\begin{array}{r}
Y(\tau)=\pi \mathrm{e}^{-c} \exp \left\{-\frac{1}{2} \int_{-\infty}^{\infty} \mathrm{d} u \ln \left[\left(u+\sqrt{u^{2}+\tau^{2}}\right)-\ln \tau\right]\right. \\
\left.\times \frac{\partial}{\partial u}\left[\left(1+x-\frac{|u-r|}{b} \frac{T}{\Delta(0)}\right) \Theta\left(x-\frac{|u-r|}{b} \frac{T}{\Delta(0)}\right) \tanh \sqrt{u^{2}+\tau^{2}}\right]\right\}
\end{array}
$$

(ii)

$$
X(\tau)=\pi \mathrm{e}^{-c} \frac{\Delta(T)}{\Delta(0)} .
$$

The formula for the critical temperature can be written as

$$
t=\frac{T_{\mathrm{c}}}{\Delta(0)}=\frac{1}{2} \exp \left\{\frac{1}{2} \int_{-\infty}^{\infty} \mathrm{d} u \ln |2 u| \frac{\partial}{\partial u}[1+(x-|u-r| c)] \tanh (u)\right\},
$$

where

$$
\frac{T_{\mathrm{c}}}{T_{\mathrm{c} 0}}=\pi \mathrm{e}^{-c} t, \quad c=\frac{t}{b} .
$$

In Figs. 6 and 7 the temperature dependences of energy gap for triangle EDOS introduced above are presented. The particular case when the maximum

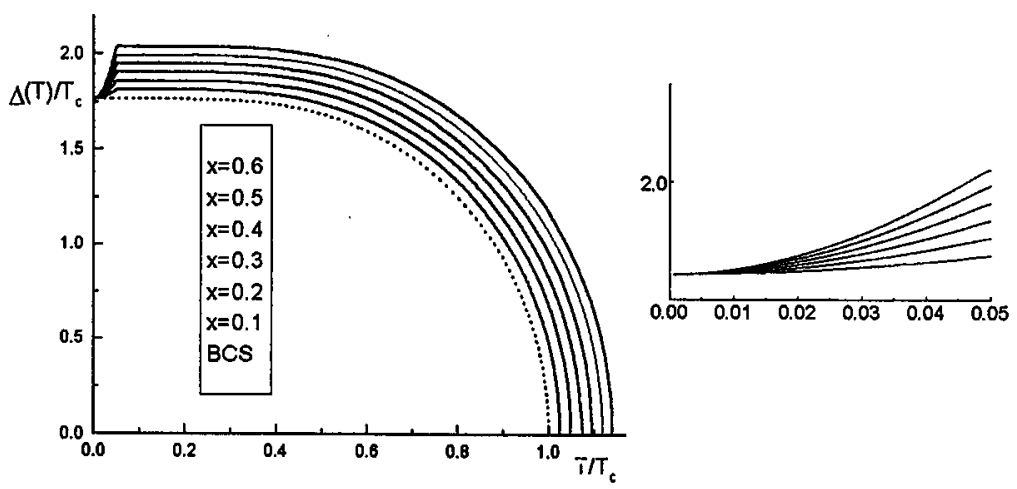

Fig. 6. The exemplary forms of the energy gap vs. reduced temperature for the triangle EDOS. Parameter $r=0$. Parameter $b$ is equal to 0.3 , and $x=0.6,0.5,0.4,0.3,0.2$. The dotted line corresponds to the BCS curve. 


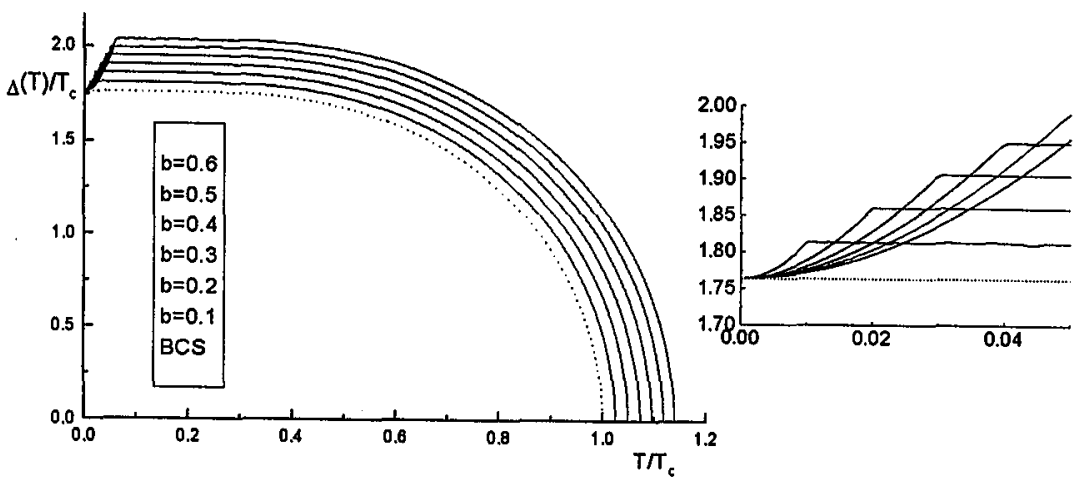

Fig. 7. The exemplary forms of the energy gap vs. reduced temperature for the triangle EDOS. Parameter $r=0$. Parameter $x$ is equal to 0.5 , and $b=0.6,0.5,0.4,0.3,0.2,0.1$. The dotted line corresponds to the BCS curve.

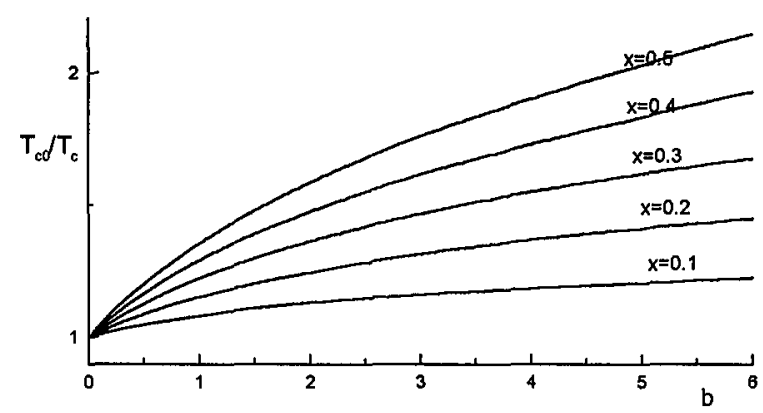

Fig. 8. Reduced critical temperature vs. $b$ parameter for the triangle EDOS. Parameter $x$ takes the values: $0.5,0.4,0.3,0.2,0.1$ and $r=0$.

of a fluctuation is localised directly on the Fermi level (which corresponds to $r=0$ ), has been considered precisely. The calculations lead to the classical BCS ratio $\Delta(0) / T_{\mathrm{c}}$ and the shape of the curves is similar to the one corresponding to the Lorentzian EDOS. One may notice that the energy gap changes more rapidly and is slightly more "edgily" near $T=0$ than in the Lorentzian case. Nevertheless, in the very narrow region when $T \rightarrow 0$, the $\Delta(T)$ curve has still a plateau, hence according to our previous discussion $C \rightarrow 0$ at $T \rightarrow 0$. Clearly, the form of a fluctuation is reflected in the behaviour of $\Delta(T)$ curve in the vicinity of $T=0$.

The critical temperatures corresponding to the triangle EDOS, for various sets of the parameters, are presented in Fig. 8. The shape of the curves is similar to the Lorentzian case. Here, the negative fluctuations have been not studied.

\section{Logarithmic EDOS}

Finally, employing the logarithmic EDOS Eq. (4) in Eq. (8), after parameterization (7) one can obtain equations convenient for numerical calculation of energy gap dependence vs. temperature in the form 
(i)

$$
Y(r)=\pi \mathrm{e}^{-c} \exp \left\{-\frac{1}{2} \int_{-\infty}^{\infty} \mathrm{d} u \ln \left[\left(u+\sqrt{u^{2}+\tau^{2}}\right)-\ln \tau\right]\right.
$$

$\left.\times \frac{\partial}{\partial u}\left[\left(1+x \ln \left(\frac{2 b}{(u-r) \frac{T}{\Delta(0)}}\right)\right) \Theta\left(\ln \left(\frac{2 b}{(u-r) \frac{T}{\Delta(0)}}\right)\right) \tanh \sqrt{u^{2}+\tau^{2}}\right]\right\}$

(ii)

$$
X(\tau)=\pi \mathrm{e}^{-c} \frac{\Delta(T)}{\Delta(0)}
$$

Consequently, in order to obtain the critical temperature we derive the formula

$$
\begin{aligned}
t= & \frac{T_{\mathrm{c}}}{\Delta(0)}=\frac{1}{2} \exp \left\{\frac{1}{2} \int_{-\infty}^{\infty} \mathrm{d} u \ln |2 u|\right. \\
& \left.\times \frac{\partial}{\partial u}\left[1+x \ln \left(\frac{2 b}{(u-r) c}\right) \Theta \ln \left(\frac{2 b}{(u-r) c}\right)\right] \tanh (u)\right\},
\end{aligned}
$$

where

$$
\frac{T_{\mathrm{c}}}{T_{\mathrm{c} 0}}=\pi \mathrm{e}^{-c} t, \quad c=b t .
$$

In Fig. 9 the temperature dependences of energy gap for the logarithmic EDOS are plotted. As above, the particular case when the fluctuation (divergence) occurs exactly on the Fermi level (which corresponds to $r=0$ ), has been investigated. We also put $x=1$, so in this case we deal only with one parameter $b$, which is roughly 10 times smaller than that considered for Lorentzian and triangle EDOS. Again, the calculations lead to the classical BCS ratio $\Delta(0) / T_{\mathrm{c}}$ and the shape of the curves is preserved. Moreover, in the very narrow region when $T \rightarrow 0, \Delta(0)$ curve has a plateau.

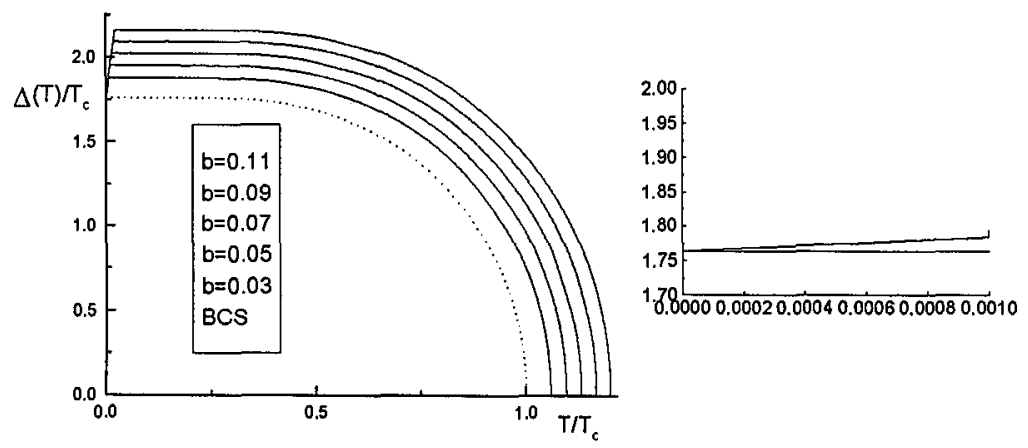

Fig. 9. The exemplary forms of the energy gap vs. reduced temperature for the logarithmic EDOS. Parameter $b$ takes the values: $0.11,0.09,0.07,0.05,0.03$, and $x=1, r=0$. The dotted line represents the BCS curve. 


\section{Energy gap behaviour near $T_{\mathrm{c}}$}

Let us notice that Eq. (6) can be symmetrised since we assume that the coupling interaction is symmetric with respect to the Fermi level. Hence, Eq. (6) may be written as

$$
1=\lambda \int_{0}^{\omega_{D}} \frac{\mathrm{d} \xi}{\sqrt{\xi^{2}+\Delta^{2}}} I(\xi) \tanh \frac{\sqrt{\xi^{2}+\Delta^{2}}}{2 T}
$$

where

$$
I(\xi)=\frac{1}{2}\left[N_{1}(\xi, B, R, x, n)+N_{1}(-\xi, B, R, x, n)\right]
$$

is the EDOS symmetric with respect to the Fermi level. For $T \rightarrow T_{\mathrm{c}}$ and $T=T_{\mathrm{c}}$ Eq. (27) reduces to the form

$$
\begin{aligned}
\frac{1}{\lambda}= & \ln \frac{\omega_{D}}{2 T}-\int_{0}^{\omega_{D} / 2 T_{\mathrm{c}}} \mathrm{d} u \ln u \frac{\mathrm{d}}{\mathrm{d} u}[I(u T) \tanh (u)] \\
& +\frac{\Delta(T)^{2}}{8 T_{\mathrm{c}}} \int_{0}^{\omega_{D} / 2 T_{\mathrm{c}}} \frac{\mathrm{d} u}{u} I\left(u T_{\mathrm{c}}\right) \frac{\mathrm{d}}{\mathrm{d} u}\left(\frac{\tanh (u)}{u}\right)
\end{aligned}
$$

and so,

$$
\frac{1}{\lambda}=\ln \frac{\omega_{D}}{2 T_{\mathrm{c}}}-\int_{0}^{\omega_{D} / 2 T_{\mathrm{c}}} \mathrm{d} u \ln u \frac{\mathrm{d}}{\mathrm{d} u}\left[I\left(u T_{\mathrm{c}}\right) \tanh (u)\right],
$$

where for large $\xi$ we have $I(\xi)=1$ and $\mathrm{d} I(\xi) / \mathrm{d} \xi=0$, as it is assumed for $N_{1}(\xi)$. Eliminating $\lambda$ and developing $I(u T)$ in a power series

$$
I(u T)=I\left(u T_{\mathrm{c}}+u\left(T-T_{\mathrm{c}}\right)\right)=I\left(u T_{\mathrm{c}}\right)+\frac{u}{T_{\mathrm{c}}}\left(T-T_{\mathrm{c}}\right) \frac{\mathrm{d}}{\mathrm{d} u} I\left(u T_{\mathrm{c}}\right)+\ldots .
$$

after some algebraic transformations one may obtain the expression for the energy gap at $T \rightarrow T_{\mathrm{c}}$ in the form

$$
\Delta(T)=2 T_{\mathrm{c}}\left[\frac{-2 \int_{0}^{\infty} \mathrm{d} u I\left(u T_{\mathrm{c}}\right) \frac{\mathrm{d}}{\mathrm{d} u} \tanh (u)}{\int_{0}^{\infty} \frac{\mathrm{d} u}{u} I\left(u T_{\mathrm{c}}\right) \frac{\mathrm{d}}{\mathrm{d} u}\left(\frac{\tanh (u)}{u}\right)}\right]^{1 / 2} \sqrt{1-\frac{T}{T_{\mathrm{c}}}}
$$

and taking into account that $I\left(u T_{\mathrm{c}}\right)=1$, we get

$$
\Delta(T)=T_{\mathrm{c}} \sqrt{\frac{8 \pi^{2}}{7 \zeta(3)}\left(1-\frac{T}{T_{\mathrm{c}}}\right)},
$$

which is just the well-known BCS formula. Equation (31) proves that any fluctuation does not change the BCS shape of the $\Delta(T)$ dependence near $T_{\mathrm{c}}$.

Our numerical results hold the BCS shape of the energy gap dependence in the $T \rightarrow T_{\mathrm{c}}$ limit. Experimental curves presented in the paper [14] remain in agreement with our conclusions. Besides, the calculations carried out by Pashitski and Pentegov [15] lead to the same results. 
On the other hand, the calculations derived for $T \rightarrow 0$ lead to the following general formula:

$$
\Delta(T)=\Delta(0)-\sqrt{2 \pi T \Delta(0)} \exp \left(-\frac{\Delta(0)}{T}\right) \frac{2}{\sqrt{\pi}} \int_{0}^{\infty} \mathrm{d} x I(x \sqrt{2 \pi T \Delta(0)}) \mathrm{e}^{-x^{2}},
$$

which confirms the presence of a plateau of the energy gap in the region close to $T=0$ with no respect to a type of the fluctuation.

\section{Conclusions}

In the paper we have studied the system with fluctuation of EDOS in the vicinity of the Fermi surface. More precisely, the fluctuation of Lotentzian, triangle, and logarithmic types has been discussed. In this formalism the total number of quasiparticles remains constant. Results of some representative numerical calculations are compared with experimental data, for instance in the papers [14, 15] the energy gap found experimentally can be fitted with the three postulated EDOS forms. It has been shown that the variation of the critical temperature depends on the fluctuation size. The shape of the fluctuation is reflected in the form of the energy gap vs. temperature dependence. Small fluctuations cause a visible change of both the critical temperature and the form of the energy gap vs. temperature plots. When the fluctuation is positive a rise of the critical temperature is observed. In turn, negative fluctuations decrease $T_{\mathrm{c}}$ resulting in the total destruction of superconductivity state in extremal cases, which seems to be an interesting property and may help to answer the question - why some materials do not reveal superconductivity? This result can be discussed in the vicinity of $T=0$, where a crucial increase or decrease in $\Delta(T)$ is noticed. This rather strange behaviour has not been observed experimentally up to now, being presumably to subtle to be measured. It could explain why for HTSCs materials the basic ratio $\Delta(0) / T_{\mathrm{c}}$ is not equal to 1.764 any more. On the other hand, in the region $T \rightarrow T_{\mathrm{c}}$ the experimental results are in satisfactory agreement with the BCS ones. In the intermediate range of temperatures an essential discrepancy with the BCS curve is observed. However, this discrepancy can be eliminated by appropriate fitting with two Lorentzian peaks.

In all cases of the fluctuations under consideration we have taken into account three groups of parameters $B_{i}, R_{i}$, and $x_{i}$, and analysed their influence on a shape of the energy gap and value of the critical temperature. However, comparing our approach to some other ones discussed in the review $[1,2]$ we can easily find experimental explanation for the parameters employed. And so, the parameter $x_{i}$, which defines the height of the fluctuation or the growth rate of the singularity, expresses the doping concentration. The parameter $B_{i}$, which is a half-width of a peak or singularity, corresponds to $1 / 2$ of the width of the singularity observed in the experiments. Moreover, what the other authors call a pseudogap is exactly $R_{i}$, i.e. the distance from the Fermi level to the peak or singularity in EDOS. So, $R_{i}$ indicates the singularity energy level in a direct manner.

The presented formalism and its connections with experimental results confirm the essential impact of fluctuations of EDOS on some amazing properties of the new generation of superconductors. We also postulate the minor influence of fluctuation on the final results in the models studied. 


\section{References}

[1] R.S. Markiewicz, J. Phys. Chem. Solids 58, 1179 (1997).

[2] J. Bouvier, J. Bok, in: The Gap Symmetry and Fluctuations in High $T_{\mathrm{c}}$ Superconductors, Eds. J. Bok, G. Deutscher, D. Pavuna, S. Wolf, Plenum Press, New York 1998, p. 37.

[3] R. Grassme, P. Seidel, J. Supercond. 9, 619 (1996).

[4] L. Force, J. Bok, Solid State Commun. 85, 975 (1993).

[5] L. Van Hove, Phys, Rev. 89, 1189 (1953).

[6] B. Mitrovic, J.P. Carbotte, Can. J. Phys. 61, 758 (1983).

[7] E. Schachinger, B. Mitrovic, J.P. Carbotte, J. Phys. F, Met. Phys. 12, 1771 (1982).

[8] K.M. Ho, M.L. Cohen, W.E. Picket, Phys. Rev. Lett. 41, 815 (1978).

[9] R. Gonczarek, M. Mulak, Phys. Lett. A 251, 262 (1999).

[10] R. Gonczarek, M. Mulak, Phys. Status Solidi $B$ 208, 457 (1998).

[11] M. Mulak, R. Gonczarek, Acta Phys. Pol. A 89, 689 (1996).

[12] R. Gonczarek, M. Mulak, W. Kumala, Acta Phys. Pol. A 95, 231 (1999).

[13] R. Gonczarek, M. Mulak, Acta Phys. Pol. A 86, 1007 (1994).

[14] H. Enomoto, H. Kokado, I. Matsubara, N. Mori, H. Ozaki, Czechoslovak J. Phys., Part S3 46, 1331 (1996).

[15] E.A. Pashitskii, V.I. Pentegov, Czechoslovak J. Phys., Part S2 46, 1001 (1996). 